\title{
Blindness in the City of Nottingham (1980-1985)
}

\author{
W. A. ACLIMANDOS and N. R. GALLOWAY \\ London and Nottingham
}

\begin{abstract}
Summary
An analysis has been made of data obtained from all available blind and partially sighted registration forms (Form BD8) for the City of Nottingham between the years 1980 and 1985 inclusive. Senile macular degeneration was the commonest cause for registration followed by glaucoma and then diabetic retinopathy. The rate of registration per 100,000 of population for different age groups is compared with similar figures for all England obtained 10 years previously. There is an apparent increase in the rate of registration of senile macular degeneration but a reduction in the rate of registration of glaucoma and cataract. The rate of registration of patients with diabetic retinopathy is also reduced but only in elderly patients
\end{abstract}

In the United Kingdom, statistical information concerning visual impairment is collected by consultant ophthalmologists who are required to complete special blind registration forms. The patients are categorised into 'blind' or 'partially sighted' according to the degree of their visual handicap. The statutory definition of blindness is that the person is 'so blind as to be unable to perform any work for which eyesight is essential'. The definition of partial sight is less clear cut but the person must be 'substantially and permanently handicapped by congenitally defective vision or from illness or injury causing defective vision'.

The procedure is entirely facultative and depends on the assessment of the consultant as well as the willingness of the patient to be registered. Unlike other countries, in the United Kingdom, registration is not legally required.

These forms are processed locally usually by the Social Services Department in order that patients who qualify can be placed on their list. Copies of blind registration forms, with names erased, are sent to the Department of Health and Social Security for computor analysis.
The aim of this study was to collect data as near to the source as possible from a relatively stable population and compare the information with that obtained ten years previously. Reliable information from the same population for those earlier years was not available and our data is compared with the figures for England from 1970-76. The population of the City of Nottingham was 279, 720 in $1984 .^{1}$

\section{Materials and Methods}

All available forms stored at the County Hall, Nottingham were examined. These totalled 701. The total figure for all new registrations for this period (1980-1985) recorded by the Visual Handicap Team was 820 (416 blind and 404 partially sighted) suggesting that a proportion of the forms had been mislaid or were missing for some other reason. Of the 701 forms examined, 629 (320 blind and 309 partially sighted) were sufficiently completed to give the required information. The number of available records of new registrations in the period from $1980^{\circ}$ to 1985 was 365 blind and 336 partially sighted.

The following information was extracted from the forms: 
(1) Date of birth

(2) Date of onset of condition leading to registration

(3) Date of registration

(4) Visual acuity in each eye

(5) Reason for registration

The figures for England 1970-1976 were obtained from Sorsby's 'Blindness and partial sight in England 1969-1976'. ${ }^{2}$ All the rates have been calculated per 100,000 of the population concerned. The total numbers are averaged per year for 1980 to 1985 in Nottingham and are compared with equivalent figures for England 1970-1976.

The aetiological assessment also shows the number per 100,000 of the population per year (1980-1985) in Nottingham and is compared to the equivalent figures for England 1970 only. Detailed aetiological figures, in particular regarding diabetic retinopathy are not complete for England 1971-1976.

\section{Results}

Figure 1 shows the number of new blind registrations per year for Nottingham City in the first half of the 1980s and for England in the first half of the 1970s. If we assume that Nottingham is not exceptional in its population or recording methods then there would appear to be a fall in the rate of blindness in the elderly. Figure 2 shows the main diagnostic categories expressed as a percentage of the total registrations from 1980-1985. Figures 3 and 6 show our own figures for the main diagnostic categories at different ages, comparing them with the all England figures obtained ten years previously. It can be seen that the apparent reduction in the incidence of blind registration can be attributed largely to a fall in the registration rate of elderly patients with glaucoma, and in the registration rate of patients with cataract. The blindness registration rate for senile macular degeneration appears to have increased. The rate of registration for elderly blind diabetics over seventy seems to have become strikingly less whereas there has been little change in those aged 65-74.

\section{Discussion}

The results can be considered a reasonably accurate analysis of the blind and partially sighted registers as $87.6 \%$ of the available blind and $91.9 \%$ of the available partially sighted BD8 forms were included in the study.

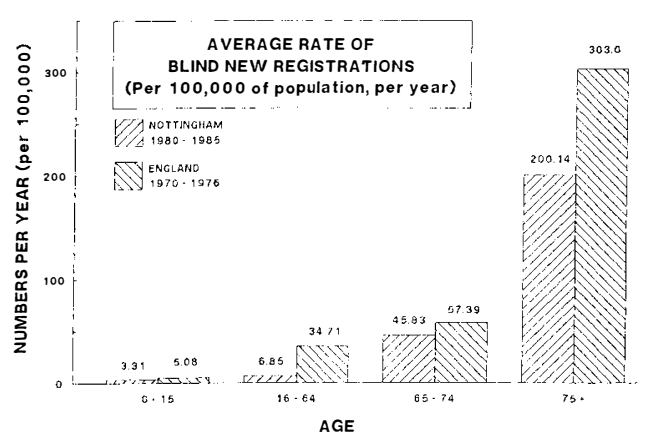

Fig. 1. Average Rate of New Blind Registrations per 100,000 .

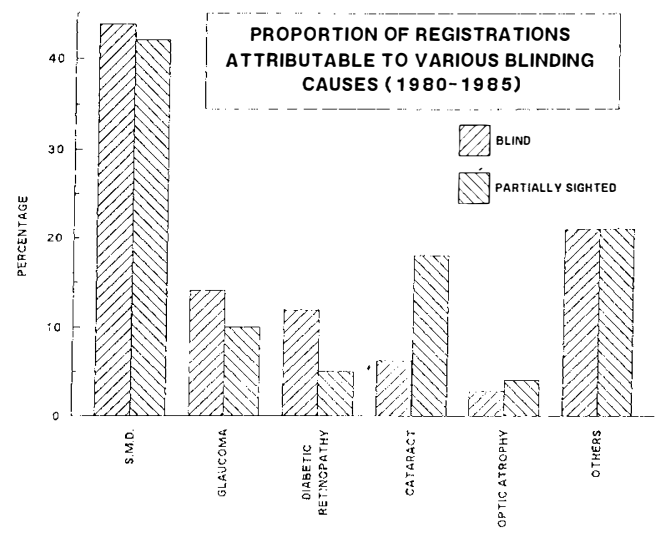

Fig. 2. Percentage of Registrations Due to Various Blinding Causes.

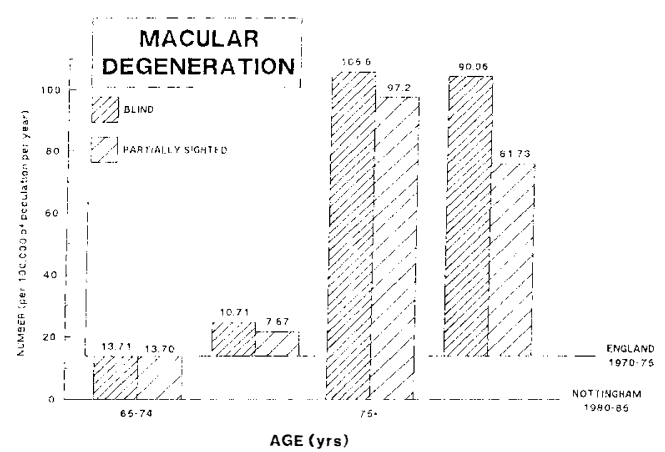

Fig. 3. Incidence per 100,000 of Blindness and Partial Sight due to Senile Macular Degeneration for different age groups. 
The number of partial sight registrations is smaller than the number of blind registrations. This probably confirms previous reports $^{3}$ suggesting that the partial sight register in particular is not a true representa-

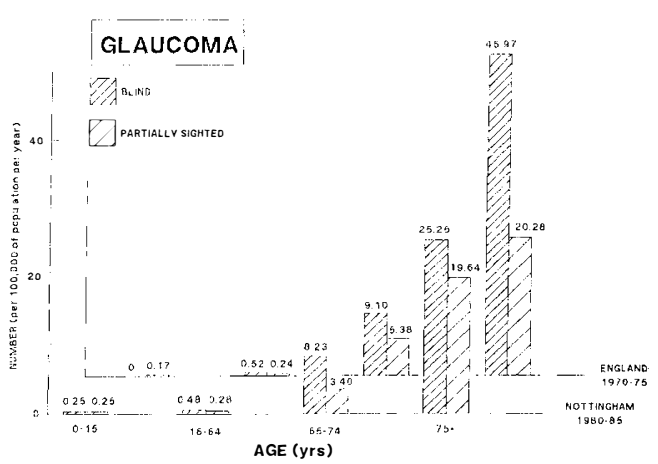

Fig. 4. Incidence per 100,000 of Blindness and Partial Sight due to Glaucoma for different age groups.

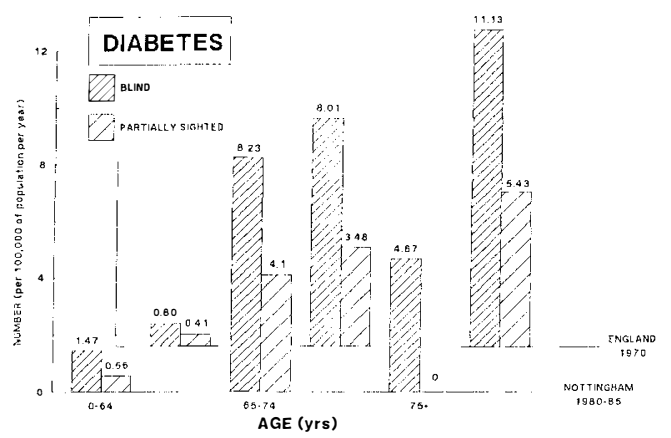

Fig. 5. Incidence per 100,000 of Diabetic Blindness and Partial Sight for different age groups.

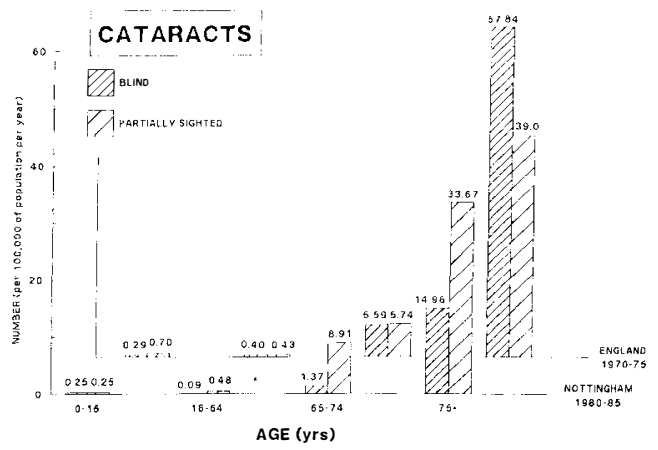

Fig. 6. Incidence per 100,000 of Blindness and Partial Sight due to Cataract for different age groups. tion of the number of visually handicapped in the population.

There are a number of factors which limit the accuracy of blind register statistics. There is undoubtedly a pool of unknown cases some of whom may have refused registration and others who have never sought attention. It would appear that the blind figures are underestimated by a factor of 1.1 and the partially sighted by a factor of $1.5 .^{4,3}$ There is a wide range of interpretations of the definitions of blindness and partial sightedness. More accurate guidelines regarding these would limit such variation. On the other hand, if the definitions become too restrictive then certain needy patients might be excluded from social support. Some BD8 forms are not completed adequately and in particular the cause of registration is not always clear. The introduction of a proper disease index as part of the written instructions attached to the form would help to solve this.

An important trend which is seen in the figures shown here, is the reduction in the rate of registration of elderly patients registered blind from glaucoma and diabetes. The rate of registration of glaucoma patients in the years $1980-1985$ seems to be reduced for all ages whereas the diabetics show a reduction in the over $75 \mathrm{~s}$ only. It seems possible that better treatment may at least have some influence here. Most analyses of blind registrations both from here and in the United States ${ }^{5,6}$ show a surprisingly high incidence of cataract. This may be because cataract may often be present together with some other untreatable cause of blindness. It may also happen that preoperative patients are placed on the blind register and then removed again after surgery. This can only distort the figures and may be of little benefit to the patients when the waiting period is short. The diagnosis on the form should be limited to the untreatable causes of blindness.

We are indebted to Mrs. Ann Padgett for her invaluable assistance in helping to gather the data and to Mrs. Pauline Shorthouse for putting the data into the computor. We would also like to thank the audio-visual department of Moorfields Eye Hospital for helping with the illustrations. 


\section{References}

1 Population Estimates for Nottinghamshire 1984 2001. Leaflet from Research and Information Unit Social Services Department. Sept. 1986.

2 Sorsby A: Blindness and Partial Sight in England 1969-1976 Rep Health Soc Subj 129, London, HMSO, 1976.

${ }^{3}$ Cullinan TR: Visually Disabled People in the Community Health Services Research Unit Report No 28. Canterbury. University of Kent.

${ }^{4}$ Gibson JM, Lavery JR, Rosenthal AR: Blind- ness and Partial Sight in an Elderly Population. Br J Ophthalmol 1986, 70: 700-5.

${ }^{5}$ Ghafour IM, Allan D, Foulds W: Common Causes of Blindness and Visual Handicap in the West of Scotland. Br J Ophthalmol 1983, 67: 209-13.

${ }^{6}$ Khan HA, Leibowitz HM, Ganley JP, Kini MM, Colton T, Nickerson RS, Dawber TR: The Framingham Eye Study 1. Outline and Major Prevalence Findings. Am J Epidemiol 1977, 106: $17-32$. 